(c) 2018 IEEE. Personal use of this material is permitted. Permission from IEEE must be obtained for all other uses, in any current or future media, including reprinting/republishing this material for advertising or promotional purposes, creating new collective works, for resale or redistribution to servers or lists, or reuse of any copyrighted component of this work in other works. 


\title{
Comparison of Reception Mechanisms for Molecular Communication via Diffusion
}

\author{
Muneer M. Al-Zu'bi, Ananda S. Mohan, and Steve S.H. Ling \\ Center for Health Technologies (CHT), Faculty of Engineering and Information Technology (FEIT) \\ University of Technology Sydney (UTS), NSW, Australia. \\ E-mail: \{muneer.al-zubi, ananda.sanagavarapu, steve.ling\}@uts.edu.au
}

\begin{abstract}
Molecular communication paradigm enables nanomachines or biological cells at nano/micro scales to communicate using chemical molecules. In this paper, we study different reception mechanisms in an unbounded 3-D biological medium for diffusion-based molecular communication system and compare their performances. The number of received molecules (i.e., number of activated receptors) is first analytically evaluated and then validated using a particle-based simulator developed by us. We address various receiver models, viz., passive, irreversible partially or fully absorptive, and a more general reversible receivers. The peak amplitude and peak time for passive and fully absorptive receivers are evaluated. The impact of various parameters, e.g., diffusion coefficient, separation distance, forward/backward reaction rates, on the received signal are examined.
\end{abstract}

Keywords-Molecular communication, nanonetwork, diffusion, nanomachine, receivers, reversible reaction.

\section{INTRODUCTION}

Nanoscale communication deals with communication between nanomachines (NMs) or nano devices at nanometer /micrometer scales [1]-[2]. Nano network is an interconnection of many nanomachines that communicate with each other in a cooperative manner [1]. Molecular communication (MC) is an emerging nano communication technology that is mainly inspired by biological mechanisms. It enables the communication between the bio-nanomachines and biological cells inside the human body using chemical molecules such ions and proteins [2]-[3]. The bio-nanomachines are tiny devices made of nano-to-micro scale components made up of biological or biocompatible materials which perform simple functions such as sensing, computation, and actuation [2].

Molecular communication (MC) offers a promising alternative for electromagnetic communication (EM) particularly for intra-body communication in microfluidic environments operating at nano/micro scales due to its biocompatibility and low energy requirements [3]. It is well known that EM communications for biological intra-body communications at this scale has some shortcomings such as requiring use of highend millimetric or terahertz wave frequency bands that could cause excessive attenuation, temperature rise and poor propagation inside the body. Among the many possible molecular transport mechanisms, molecular communication via diffusion (MCvD) is the most simple method of molecular transport within biological fluidic microenvironments which does not require extra external energy nor any communication infrastructure [2]-[4]. Thus, it is a well suited method of communication for many biomedical applications such as targeted drug delivery, in-body nanonetworks, lab-on-chip systems, etc [5]. In MCvD, the information molecules diffuse randomly according to Brownian dynamics which can be mathematically modelled using Fick's laws of diffusion [6, 7]. In this random motion, the molecules propagate by utilizing the thermal energy that is already present in the fluidic environment. Thus, no source of external energy is required for diffusion-based biological molecular communications.

In the literature, many works on molecular communication (MC) are available. A new physical end-to-end model for molecular communication is presented by modeling the emission, diffusion, and reception processes in [8] where the normalized gain and delay are evaluated as a function of the input frequency and transmission range. An energy model for molecular communication via diffusion (MCvD) is developed in [9] which has been optimized based on channel capacity and data rate. Optimized design models for passive receiver in a diffusion-based molecular communication channel are also available [10]-[11] which include the flow, noise, and enzymes present in fluidic propagation micro-environments. In [12], analytical expressions for communication metrics, viz., pulse delay, pulse amplitude and pulse width, are derived for MCvD system using amplitude and energy detection techniques while in [13], closed-form expressions are derived for error probability using these detection techniques. Also, analytical expression for fraction of molecules absorbed by an absorbing receiver in MCvD system is provided in [14]. The effect of interference on a target receiver has also been examined using Bit Error Rate (BER) and capacity [15]. A statistical-physical model for the interference in diffusion-based molecular nano networks due to molecules that are simultaneously emitted by multiple transmitting nanomachines is available in [16]. In [17], an equivalent discrete-time channel model is derived for molecular communication via diffusion based on the characteristic function with emphasis on an absorbing receiver.

Inside a biological medium, when a transmitter nano machine releases the information molecules, they reach a targeted receiver cell and may react with the protein receptors lying on its surface. Then, the information molecules may bind to the receptors to activate them. The number of activated receptors (ligand-receptor complexes) that create a chemical reaction inside the cells after reaching a specific threshold 
forms the received molecular signal. There are many receiver models available for $\mathrm{MCvD}$ such as passive, irreversible partially or fully absorptive, and more general reversible receivers. In [18]-[19], an analytical reversible receiver models were provided along with an expression for the expected number of absorbed molecules i.e., number of the activated receptors.

In this paper, we compare the different types of receiver models for MCvD in terms of the expected number of received molecules and other communication metrics, viz., peak amplitude and peak time, using both analytical and simulation approaches. We have developed a particle based simulator to obtain the expected number of the received molecules at any receiver nanomachines $(\mathrm{RN})$ and compare with the results obtained using analytical expressions provided in the literature. We consider here passive, irreversible partially or fully absorptive, and a more general reversible receivers.

This paper is organized as follows. Firstly, we introduce the system model and theoretical aspects of diffusion and various types of RN in section II. In section III, the analytical and simulation results are provided with a discussion. Finally, the conclusions will be presented in section IV.

\section{SYSTEM MODEL}

In this section, the various reception mechanisms will be demonstrated and the expressions for expected number of received molecules will be presented for MCvD system. In this model, we assume there is a transmitting nano machine (TN) that seeks to communicate with a receiving nano machine (RN) in a 3-D unbounded diffusive molecular fluidic environment as shown in Fig. 1. The $\mathrm{TN}$ is a point source that encodes the information on the molecule concentration, which can be considered as" Concentration Shift Keying (CSK)". It emits the molecules instantaneously into the diffusive fluidic medium, which acts as the propagating channel. The size of the $\mathrm{TN}$ is assumed negligible compared to the relative distance between $\mathrm{TN}$ and $\mathrm{RN}$.

Once released, the information molecules diffuse randomly in all directions throughout the fluidic environment following Brownian motion. The biological fluidic environment such as blood vessels etc., act as the propagation medium (channel) for molecular diffusion. We assume the molecular collisions with the medium boundaries to be elastic i.e., the environment to be unbounded. A fraction of the originally emitted molecules may reach a targeted cell i.e., a receiver (RN) via diffusion. If the $\mathrm{RN}$ is a passive receiver, then the molecules that reach will be counted without any absorption. If the $\mathrm{RN}$ is a generalized reversible receiver, there will be a reaction at its surface. The number or received molecules over time at the RN represent the received signal amplitude or the channel impulse response (CIR).

Fick's second law of diffusion [7] characterizes the diffusion of molecules mathematically in a fluidic environment as [11]

$$
\frac{\partial C(x, y, z, t)}{\partial t}=D \nabla^{2} C(x, y, z, t)
$$

where, $\nabla^{2}$ is Laplacian operator and $C(x, y, z, t)$ is the spatiotemporal distribution of information molecules at any point $(x, y, z)$ and at time $t$.

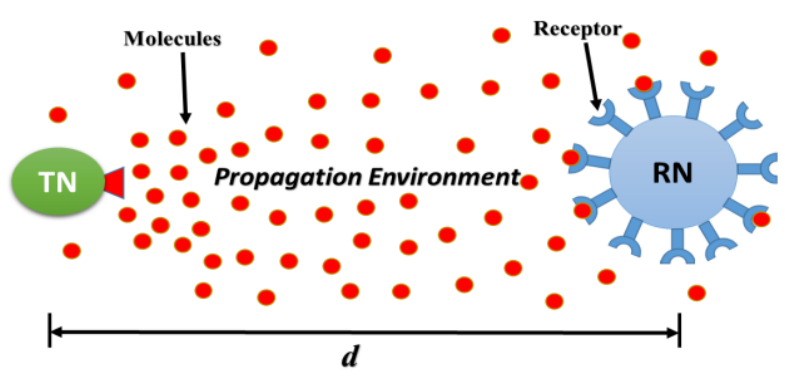

Fig. 1. Graphical representation of molecular communication via diffusion system.

The diffusion coefficient $(D)$ of the fluid environment is given by [10]

$$
D=\frac{K_{B} T_{a}}{6 \pi \eta r_{m}}
$$

where, $K_{B}$ is the Boltzmann constant, $T_{a}$ is the absolute temperature in (Kelvin), $\eta$ is the dynamic viscosity of the fluid environment, and $r_{m}$ is the Stoke's radius of the information molecules. In this work, we assume that the diffusion to be isotropic meaning the diffusion coefficient to be constant and uniform in all the directions.

If a cell acting as a receiver ( $\mathrm{RN}$ ) is a passive receiver, its presence inside a 3-D fluidic environment will not affect the diffusion process of the released molecules, and hence they can pass through the boundaries of the receiver without any physical or chemical reactions. Thus, the channel impulse response at a passive $\mathrm{RN}$ that is located at any arbitrary location $(x, y, z)$ due to a point transmitter $\mathrm{TN}$ placed at $\left(x_{0}, y_{0}, z_{0}\right)$ releasing the information molecules instantaneously into the 3-D fluidic channel can be written as [12]

$$
h(x, y, z, t)=\frac{1}{(4 \pi D t)^{3 / 2}} \exp \left(-\frac{d^{2}}{4 D t}\right), \quad t \geq 0
$$

where, $d$ is the separation distance between $\mathrm{TN}$ and $\mathrm{RN}$ given by $d^{2}=\left(x-x_{0}\right)^{2}+\left(y-y_{0}\right)^{2}+\left(z-z_{0}\right)^{2}$.

Equation (3) represents the solution of the diffusion equation (1) with impulsive emission of molecules as the initial condition and elastic boundary collisions of molecules as the boundary condition.

In general, the total number of received molecules at a passive $\mathrm{RN}$ can be calculated by first integrating the channel impulse response over the volume of $\mathrm{RN}$ and then multiplying 
the result by the initial strength of the source (i.e., total number of released molecules at time $\mathrm{t}=0$ ) [20]. Under the assumption that the distance between $\mathrm{TN}$ and $\mathrm{RN}$ is large compared to the size of the receiver, the random molecular concentration inside the volume of a receiver will be uniformly distributed. Thus, the number of received molecules by a passive $\mathrm{RN}$ can be expressed as

$$
N_{P}=Q V_{r x} h(x, y, z, t)
$$

where $\mathrm{Q}$ is the number of released molecules at time $t=0, V_{r x}$ is the volume of spherical $\mathrm{RN}$ given as $V_{r x}=4 \pi / 3 R_{r x}^{3}$, and $R_{r x}$ is the radius of the receiver.

In general, the information molecules react with protein receptors that are present on the surface of an $\mathrm{RN}$ via second order reversible reaction mechanism. Thus, they may either activate or deactivate the receptors by either binding or unbinding to the receptors, respectively. Moreover, the molecules may reflect back into the fluidic environment after colliding with the receiver surface. Therefore, the presence of a reversible $\mathrm{RN}$ will have measurable impact on the channel impulse response.

In a reversible receiver, the reaction process that the molecules undergo with the receptors at the receiver surface is reversible [19]. Hence, for a reversible receiver, the channel impulse response provides the fraction of received molecules or activated receptors until the time $t$ which is given in [19, Eq. (29)] and can be evaluated numerically. However, for an irreversible receiver, the receptors will not be deactivated after forming the ligand-receptor complexes and thus the molecules will not be dissociated from the receiver surface. The fraction of received molecules (or activated receptors) by an irreversible receiver until the time $t$ can be expressed as [18. Eq. (21)],

$$
\begin{array}{r}
N_{I}=\frac{R_{r x} K_{f}-1}{d K_{f}}\left[1+\operatorname{erf}\left(\frac{R_{r x}-d}{\sqrt{4 D t}}\right)-\exp \left(\left(d-R_{r x}\right) K_{f}+D t K_{f}^{2}\right)\right. \\
\left.\quad \times \operatorname{erfc}\left(\frac{d-R_{r x}+2 D t K_{f}}{\sqrt{4 D t}}\right)\right]
\end{array}
$$

Where, $K_{f}$ is the forward reaction rate in $(\mathrm{m} / \mathrm{s})$.

If the $\mathrm{RN}$ is a fully absorptive receiver, then all the molecules will be absorbed as soon as they collide with the surface of the receiver. For a fully absorptive RN, the fraction of received molecules until time $t$ can be obtained as [14]

$$
N_{A}=\frac{R_{r x}}{d} \operatorname{erfc}\left(\frac{d-R_{r x}}{\sqrt{4 D t}}\right)
$$

where, $\operatorname{erfc}($.$) is the complementary error function.$

Now, the expected number of received molecules during a sample duration $[\Delta t, t+\Delta t]$ can be expressed as

$$
\bar{N}(t, t+\Delta t)=Q[N(t+\Delta t)-N(t)]
$$

where, $N(t)$ is the number of received molecules until time $t$ which is obtained using either [19, Eq. (29)], (5), or (6).

The signal peak time is the time instant at which the signal has the maximum amplitude. For example, Fig. 2 shows the number of received molecules by passive $\mathrm{RN}$ as a function of time using both simulation and analytical expressions given by (4). The peak amplitude and peak time are indicated in this figure.

The peak time for the passive $\mathrm{RN}$ case can be obtained by finding the time instant at which the time derivative of (3) vanishes to get [12]

$$
t_{\max }=\frac{d^{2}}{6 D}
$$

Then, by substituting (8) in (4), we get an expression for peak amplitude [12] as

$$
N_{\max }=\frac{Q V_{r x}}{d^{3}}\left(\frac{3}{2 \pi e}\right)^{3 / 2}
$$

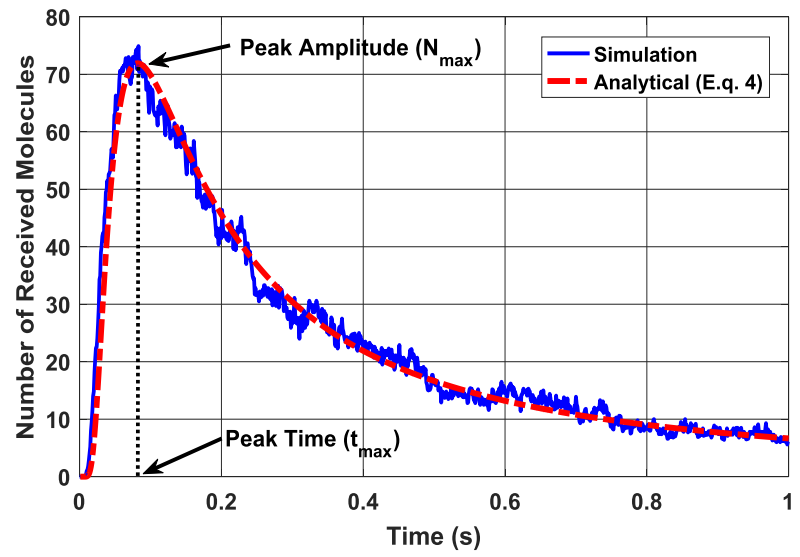

Fig. 2. Number of received molecules vs. time for a passive receiver using the system parameters listed in Table I.

For a fully absorptive receiver, the peak time and peak amplitude can be derived as [14],

$$
\begin{gathered}
t_{\text {max }}=\frac{\left(d-R_{r x}\right)^{2}}{6 D} \\
N_{\max }=\frac{Q D R_{r x} \Delta t}{d\left(d-R_{r x}\right)^{2}} \frac{e^{-3 / 2}}{\sqrt{\pi / 54}}
\end{gathered}
$$

However, for both the reversible and irreversible receivers, the peak time and peak amplitude can be evaluated numerically by finding the maximum amplitude of the received signal and identifying the corresponding time instant, respectively. 


\section{RESULTS}

In this section, we compare the various receiver models and corresponding reception mechanisms for diffusion-based molecular communication systems by using both analytical and simulation approaches. We evaluate the received signal and the two most important communication metrics, viz., peak amplitude and peak time for passive, reversible, and irreversible receivers using the analytical expressions given in section II. The analytical results have also been verified with simulation results which are obtained by our particle-based simulator using the parameters that are listed in table I. It can be seen that both the results agree well. Fig. 3 shows the peak amplitude and peak time for fully absorptive receiver as a function of the separation distance between $\mathrm{TN}$ and $\mathrm{RN}$ for various values of the diffusion coefficients, which are obtained using Eqs. (10)-(11). The peak amplitude is found to decrease (with a corresponding increase in the peak time) with increasing the $\mathrm{TN}$ and $\mathrm{RN}$ separation. This due to the longer diffusion time taken by molecules to reach the $\mathrm{RN}$ that is located far from the TN. Thus number of the molecules that successfully reach the receiver will decrease following an inverse cubic law i.e., inversely proportional to the cube of the distance i.e., $N_{\max } \sim 1 / d^{3}$ as can be seen in (9) and (11).

Table I. Simulation Parameters.

\begin{tabular}{c|c}
\hline Parameter & Value \\
\hline Simulation time $(T)$ & $1 \mathrm{~s}$ \\
\hline Time step $(\Delta t)$ & $0.1 \mathrm{~ms}$ \\
\hline Number of emitted molecules & 10,000 \\
\hline Diffusion coefficient $(D)$ & $100 \mu \mathrm{m}^{2} / \mathrm{s}$ \\
\hline Radius of RN $\left(R_{r x}\right)$ & $2 \mu \mathrm{m}$ \\
\hline Distance between TN and RN & $7 \mu m$ \\
\hline Forward Reaction constant $\left(K_{f}\right)$ & $\{50,100, \infty\} \mu \mathrm{m} / \mathrm{s}$ \\
\hline Backward Reaction constant $\left(K_{b}\right)$ & $\{0,1,3\} \mathrm{s}^{-1}$ \\
\hline Number of iterations & 100 \\
\hline
\end{tabular}

Here, it is worth noting that the contrast between molecular communication via diffusion and wireless communications using electromagnetic waves. Unlike the molecular communication, in wireless communication operating in free space, the magnitude of the power of the received signal is inversely proportional to the square of the distance, i.e., $P \sim 1 / \mathrm{d}^{2}$. But in molecular communication via diffusion, that the increase in TN-RN separation distance produces higher attenuation of the received signal. The peak time is proportional to the square of the separation distance between TN and RN, i.e., $t_{\max } \sim d^{2}$, as can be seen in (8) and (10).

Moreover, the peak amplitude increases as the diffusion coefficient increases due to faster diffusion of the molecules but with the corresponding decrease in the peak time. Therefore, the number of molecules that reach the RN will increase and thus the peak amplitude increases.
The cumulative number of the received molecules vs. time for various reversible receiver models including irreversible (fully and partially absorptive) receivers is shown in Fig. 4. The results in this figure are plotted for different values of forward $\left(K_{f}\right)$ and backward $\left(K_{b}\right)$ reaction constants. The simulations results are obtained using our particle-based simulator and one can observe good match with the analytical results. The analytical results for the irreversible receiver are evaluated using [19, Eq. (29)] by transforming all the parameters into a dimensionless form.

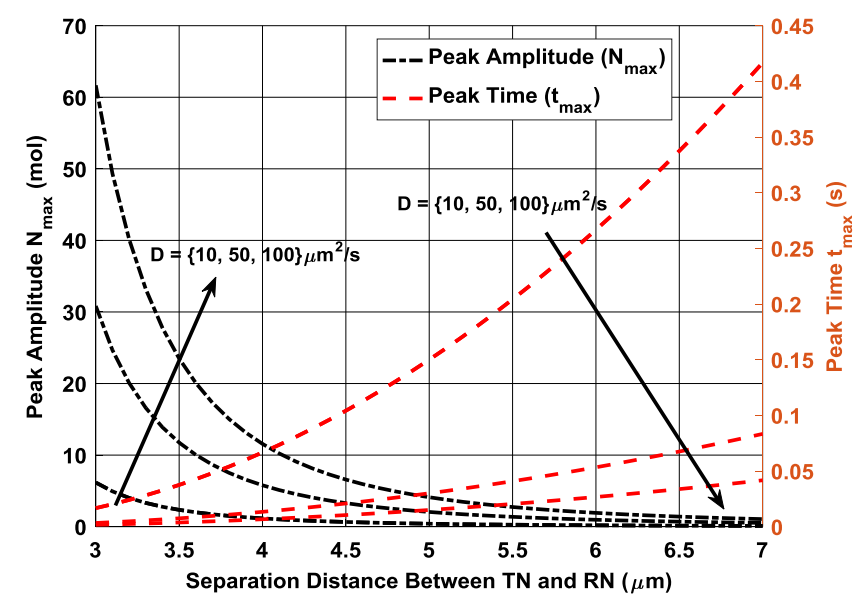

Fig. 3. Peak amplitude and peak time of fully absorbing RN vs. separation distance between $\mathrm{TN}$ and $\mathrm{RN}$ for various values of the diffusion coefficient.

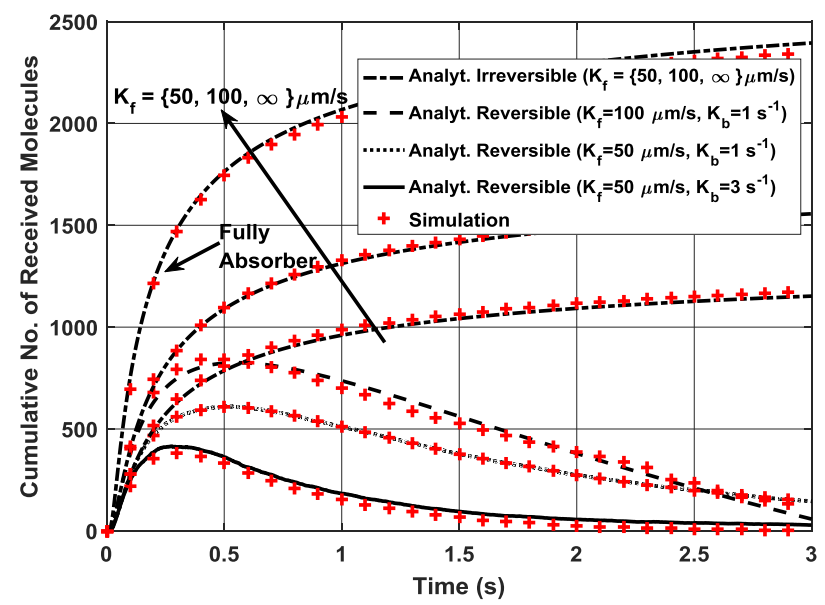

Fig. 4. The cumulative number of received molecules as a function of time for various receiver models.

An irreversible receiver model is a special case of reversible receiver with zero backward reaction constant (i.e., $K_{b}=0$ ). The fully absorptive receiver is an irreversible receiver with very large forward reaction constant $\left(K_{f} \rightarrow \infty\right)$ which has the higher amplitude compared to other receiver models. Here, all the molecules that collide with the receiver surface will be absorbed and thus the cumulative number of received molecules continues to increase until an equilibrium state (steady state) is reached. For an irreversible receiver, as the forward reaction rate decreases, the amplitude of the received 
signal decreases due to fact that some of the molecules which collide with the receiver surface will reflect back to the channel and will not be absorbed. However, the amplitude of the received signal for the reversible receiver will decrease after some time as the backward reaction constant increases. This is due to the increase in unbinding of molecules from the receptors with resultant decrease in the number of the activated receptors.

\section{CONCLUSIONS}

In this paper, we compare the molecular received signal for various receiver ( $\mathrm{RN})$ models, viz., passive, reversible, and irreversible (partially absorptive and fully absorptive) receivers. We present analytical expressions for channel impulse response, peak time, and peak amplitude for various $\mathrm{RN}$ models from the literature. These analytical expressions are compared with our simulations that show perfect match.

The peak amplitude of a passive receiver does not depend on the diffusion coefficient. For the fully absorptive receiver, the peak amplitude depends linearly on the diffusion coefficient. However, the peak amplitude is inversely proportional to the third power of the separation distance between $\mathrm{TN}$ and $\mathrm{RN}$ for both passive and fully absorptive RNs. The peak time is proportional to the squared distance and inversely proportional to the diffusion coefficient for both passive and fully absorptive receivers. The fully absorptive receiver gives the higher number of received molecules than other receiver models. Moreover, as the forward reaction rate increases, the number of activated receptors increases. But, if the backward reaction rate increases, there is a corresponding decrease in the number of activated receptors due to effect of the reversibly unbound molecules.

\section{REFERENCES}

[1] I. F. Akyildiz, F. Brunetti, and C. Blázquez, "Nanonetworks: A new communication paradigm," Computer Networks, vol. 52, pp. 2260-2279, 2008.

[2] T. Nakano, A. W. Eckford, and T. Haraguchi, Molecular communication: Cambridge University Press, 2013.

[3] N. Farsad, H. B. Yilmaz, A. Eckford, C.-B. Chae, and W. Guo, "A comprehensive survey of recent advancements in molecular communication," IEEE Communications Surveys \& Tutorials, vol. 18, pp. 1887-1919, 2016.

[4] P.-C. Yeh, K.-C. Chen, Y.-C. Lee, L.-S. Meng, P.-J. Shih, P.-Y. Ko, et $a l .$, "A new frontier of wireless communication theory: diffusion-based molecular communications," IEEE Wireless Communications, vol. 19, 2012.

[5] T. Nakano, M. J. Moore, F. Wei, A. V. Vasilakos, and J. Shuai, "Molecular communication and networking: Opportunities and challenges," IEEE transactions on nanobioscience, vol. 11, pp. 135-148, 2012.

[6] H. C. Berg, Random walks in biology: Princeton University Press, 1993.

[7] J. Crank, The mathematics of diffusion: Oxford university press, 1979.

[8] M. Pierobon and I. F. Akyildiz, "A physical end-to-end model for molecular communication in nanonetworks," IEEE Journal on Selected Areas in Communications, vol. 28, 2010.

[9] M. Ş. Kuran, H. B. Yilmaz, T. Tugcu, and B. Özerman, "Energy model for communication via diffusion in nanonetworks," Nano Communication Networks, vol. 1, pp. 86-95, 2010.

[10] A. Noel, K. C. Cheung, and R. Schober, "Optimal receiver design for diffusive molecular communication with flow and additive noise," IEEE transactions on nanobioscience, vol. 13, pp. 350-362, 2014.
[11] D. Kilinc and O. B. Akan, "Receiver design for molecular communication," IEEE Journal on Selected Areas in Communications, vol. 31, pp. 705-714, 2013.

[12] I. Llatser, A. Cabellos-Aparicio, M. Pierobon, and E. Alarcón, "Detection techniques for diffusion-based molecular communication," IEEE Journal on Selected Areas in Communications, vol. 31, pp. 726$734,2013$.

[13] A. Aijaz and A.-H. Aghvami, "Error performance of diffusion-based molecular communication using pulse-based modulation," IEEE transactions on nanobioscience, vol. 14, pp. 146-151, 2015.

[14] H. B. Yilmaz, A. C. Heren, T. Tugcu, and C.-B. Chae, "Threedimensional channel characteristics for molecular communications with an absorbing receiver," IEEE Communications Letters, vol. 18, pp. 929932, 2014.

[15] Y. Lu, M. D. Higgins, A. Noel, M. S. Leeson, and Y. Chen, "The effect of two receivers on broadcast molecular communication systems," IEEE transactions on nanobioscience, vol. 15, pp. 891-900, 2016.

[16] M. Pierobon and I. F. Akyildiz, "A statistical-physical model of interference in diffusion-based molecular nanonetworks," IEEE Transactions on communications, vol. 62, pp. 2085-2095, 2014.

[17] M. Damrath, S. Korte, and P. A. Hoeher, "Equivalent discrete-time channel modeling for molecular communication with emphasize on an absorbing receiver," IEEE transactions on nanobioscience, vol. 16, pp. 60-68, 2017.

[18] Y. Deng, A. Noel, M. Elkashlan, A. Nallanathan, and K. C. Cheung, "Modeling and simulation of molecular communication systems with a reversible adsorption receiver," IEEE Transactions on Molecular, Biological and Multi-Scale Communications, vol. 1, pp. 347-362, 2015.

[19] A. Ahmadzadeh, H. Arjmandi, A. Burkovski, and R. Schober, "Comprehensive reactive receiver modeling for diffusive molecular communication systems: Reversible binding, molecule degradation, and finite number of receptors," IEEE transactions on nanobioscience, vol. 15, pp. 713-727, 2016.

[20] V. Jamali, A. Ahmadzadeh, C. Jardin, H. Sticht, and R. Schober, "Channel estimation for diffusive molecular communications," IEEE Transactions on Communications, vol. 64, pp. 4238-4252, 2016. 